\title{
Latest Developments in Environmental SEM Systems
}

\author{
Ernst Jan R. Vesseur ${ }^{1}$
}

${ }^{1}$ Thermo Fisher Scientific, Materials \& Structural Analysis, Eindhoven, the Netherlands.

The study of materials at high resolution and in their natural state (hot, cold, dirty, wet, dry or reactive) is enabled by environmental scanning electron microscopy (ESEM) [1]. ESEM is uniquely positioned to probe the surface of materials, in-situ, at length scales that no other technique can address. During the past couple of decades, ESEM has stimulated research on wetting [2], oxidation and corrosion [3], reduction [4], etching, materials growth [5], catalysis and more.

In this paper, we present the latest developments on our ESEM products, and show how we have added new options for detection and automation, how we better accommodate analytics, and how the systems are easier to use. Last but not least, we present new sample heating options that complement and extend the in-situ capabilities. With these sample heating options, we can access conditions that are more extreme, with more precise control, and with better image quality, opening up new applications for ESEM and in-situ experimentation.

As required by multi-user facilities, the latest Thermo Scientific ESEM systems are an excellent platform for microanalysis. The chamber provides ample room for analytical detectors, including $180^{\circ}$ dual EDS, coplanar EDS and EBSD, and parallel beam WDS. The analytical capabilities are now complemented by an optional red-green-blue cathodoluminescence (CL) detector, providing color images highlighting sample properties that are not visible with the other techniques. The detector has a retractable design, does not require optical alignments, allows for a range of working distances, does not limit the field of view and is integrated in the user interface, making it highly flexible and easy to use.

Software innovations include ease of use improvements, such as undo/redo functionality, and a help function (User Guidance) that not only instructs, but also directly interacts with the microscope. A new package, AutoScript 4, provides the automation required to enable unattended data acquisition, with user-programmed imaging and stage movements. AutoScript 4 is Python-based, allowing access to a huge library of advanced functions.

Finally, a unique range of sample heating capabilities improves the support for in-situ experimentation. Samples can be heated in high vacuum (for a clean surface), in ESEM mode (for outgassing materials and for chemical reactions such as oxidation), or with extreme temperature ramp rates (for small samples) using three specialized, software integrated heating stage designs.

The new High Vacuum Heating Stage heats up to $1100^{\circ} \mathrm{C}$ while remaining in high vacuum - ideal to study crystal dynamics on clean surfaces. High vacuum also allows for excellent imaging quality and speed, enabling the rapid imaging of dynamic particles at high temperature. This heating stage is compatible with EBSD, which can be carried out with sample temperatures up to $900^{\circ} \mathrm{C}$. The second heater type is the conventional ESEM heating stage, which allows sample temperatures up to $1400^{\circ} \mathrm{C}$ and reactions with the gas that is fed into the chamber. Finally, the so-called $\mu$ Heater [6] heats a $100 \mu \mathrm{m}$ diameter area up to $1200^{\circ} \mathrm{C}$ in less than 0.1 second. These extreme ramp rates $\left(>10^{4}{ }^{\circ} \mathrm{C} /\right.$ second $)$ come with very minimal sample drift, allowing studies of nanoparticles in conditions previously not accessible. Moreover, the $\mu$ Heater is compatible with analytics (EDS, EBSD). These heating stages open 
up many new applications for ESEM and in-situ heating.

In conclusion, support for ESEM is maintained and improved on a very complete analytical platform, which includes a new cathodoluminescence detector option, the widest range of sample heating options and new possibilities for automation and ease of use.

\section{References:}

[1] D.J. Stokes, "Principles and practice of variable pressure/environmental scanning electron microscopy (VP-ESEM)", John Wiley \& Sons (2008)

[2] O. Goldbart et al, PNAS 113 (2016) p. 13624

[3] T. Jonsson et al, Corrosion Science 51 (2009) p. 1914

[4] M. Kolíbal, et al, Nano Lett. 16(8) (2016), p. 4880

[5] Z.-J. Wang et al, Nature Communications 7 (2016) p. 13256

[6] L. Novák et al, Microsc. Microanal. 23 (Suppl. S1) (2017), p. 928

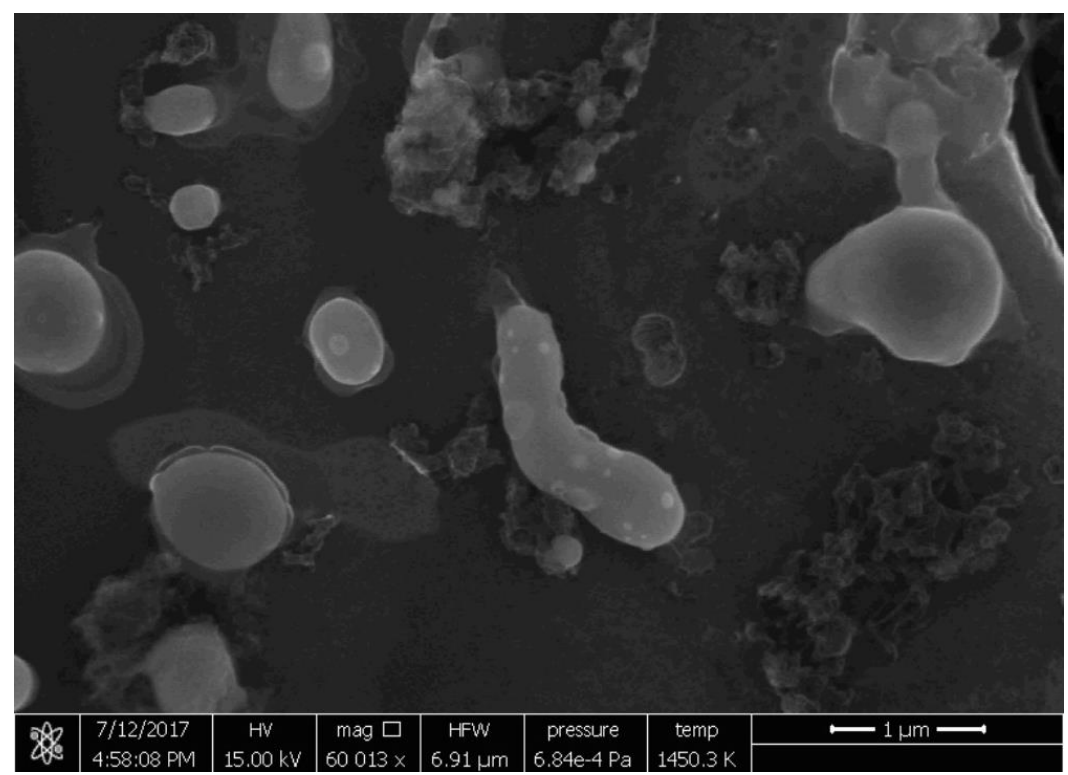

Figure 1. Video still: mix of $\mathrm{Sn}, \mathrm{Cu}, \mathrm{Ag}$ nanoparticles heated from $477^{\circ}$ to $1200^{\circ} \mathrm{C}$ in 20 seconds with minimal sample drift using the $\mu$ Heater. Image courtesy: Min $\mathrm{Wu}$, Thermo Fisher Brno

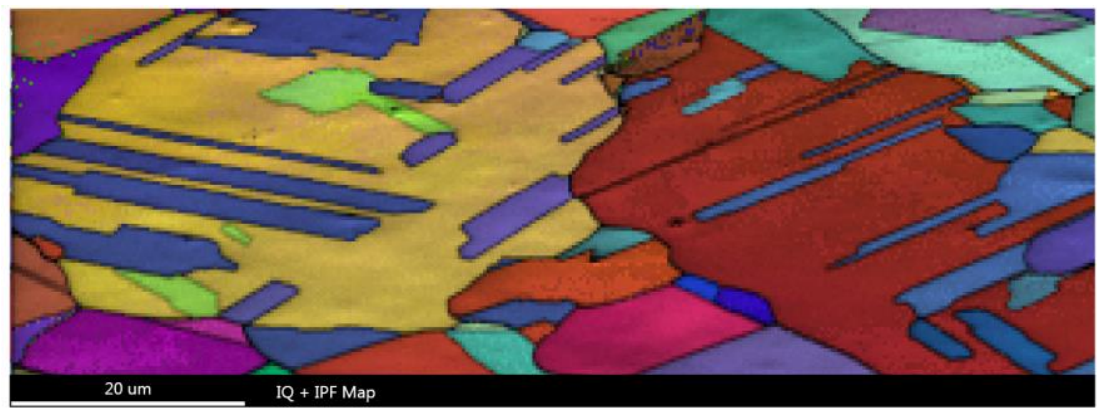

Figure 2. EBSD map acquired on a copper surface at $600^{\circ} \mathrm{C}$ in 4 minutes using the High Vacuum Heating Stage. Image courtesy: Petr Wandrol, Thermo Fisher Brno 